\title{
Efecto in vitro de Bacillus thuringiensis sobre la liberación de huevos por proglótidos de Taenia pisiformis
}

\author{
In vitro effect of Bacillus thuringiensis on the eggs release by proglottids of Taenia pisiformis \\ Emmanuel Dunstand-Guzmán ${ }^{1}$ (D) , Claudia Hallal-Calleros ${ }^{2}$ (D) , Laura Patricia Lina-García ${ }^{2}$ (D) , \\ Víctor Manuel Hernández-Velázquez ${ }^{1}$ (D) , Andrea Valeria Vega-Salazar ${ }^{1}$ (D) , \\ Fernando Iván Flores-Pérez ${ }^{2^{*}}$ (D) , Guadalupe Peña-Chora ${ }^{3^{*}}$
}

${ }^{1}$ Centro de Investigación en Biotecnología, Universidad Autónoma del Estado de Morelos (UAEM), Av. Universidad 1001, Col. Chamilpa, 62209, Cuernavaca,
Morelos, México.
${ }^{2}$ Facultad de Ciencias Agropecuarias (UAEM).
${ }^{3}$ Centro de Investigaciones Biológicas (UAEM).

*Autor para correspondencia: penachora@uaem.mx, ivan.flores@uaem.mx

Fecha de recepción:

24 de junio de 2020

Fecha de aceptación:

27 de mayo de 2021

Disponible en línea:

24 de septiembre de 2021

Este es un artículo en acceso

abierto que se distribuye de acuerdo a los términos de la licencia Creative Commons.

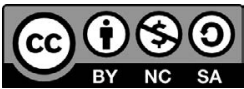

Reconocimiento-

NoComercia-

CompartirIgual 4.0

Internacional

\section{RESUMEN}

La cepa GP526 de Bacillus thuringiensis Berliner es letal para los estadios de huevo y adulto de Ancylostoma caninum Ercolani y Dipylidium caninum Linneo. Debido a su potente acción antihelmíntica, el objetivo del presente estudio fue evaluar los efectos de la cepa GP526 de manera in vitro en los proglótidos de Taenia pisiformis Bloch. Se obtuvieron proglótidos grávidos que se incubaron por $0.5,1,1.5,2$ y $2.5 \mathrm{~h}$ con el complejo espora-cristal de GP526. Los resultados indicaron que a 1.5 h post-incubación con GP526, se disminuyó la liberación de huevos vs el grupo control $(\mathrm{P}<0.001)$; además, GP526 indujo una contracción del tegumento que rodea al poro genital. Tomando en cuenta lo anterior, proponemos la cepa GP526 como un candidato para reducir la capacidad infectiva de T. pisiformis e interrumpir su ciclo biológico.

\section{PALABRAS CLAVE}

Antihelmíntico, teniasis, control biológico, céstodo, proglótidos.

\section{ABSTRACT}

Bacillus thuringiensis Berliner GP526 strain is lethal to the egg and adult stages of Ancylostoma caninum Ercolani y Dipylidium caninum Linneo. As it has a powerful anthelmintic action, the objective of the present study was to evaluate the effects of the GP526 strain in vitro on the proglottids of Taenia pisiformis Bloch. Gravid proglottids were obtained, they were incubated at 0.5, 1, 1.5, 2 and $2.5 \mathrm{~h}$ with the GP526 spore-crystal complex. The results indicated that at $1.5 \mathrm{~h}$ post-incubation with GP526, the release of eggs decreases in comparison to the control group $(\mathrm{P}<0.001)$. Moreover, a contraction of the tegument surrounding the genital pore was observed. We propose the GP526 strain as an effective candidate to reduce the infectivity of T. pisiformis tapeworm and to interrupt its biological cycle.

\section{KEYWORDS}

Antihelminthic, teniasis, biological control, cestode, proglottids. 


\section{INTRODUCCIÓN}

El céstodo Taenia pisiformis Bloch es un parásito de cánidos silvestres y domésticos que, en su fase de metacéstodo, afecta a lagomorfos como conejos y liebres. El consumo de los huevos del parásito por parte del conejo causa la cisticercosis cunícola (Loos-Frank 2000), y cada proglótido grávido de T. pisiformis es capaz de producir 41,000 huevos en promedio (Coman y Rickard 1975). Un perro infectado por un solo céstodo puede arrojar en materia fecal aproximadamente 250,000 huevos al día (DeWolf et al. 2014), por lo que resulta una infección con alta incidencia en conejos silvestres. La cisticercosis por T. pisiformis en conejos silvestres presenta mayor frecuencia en hembras respecto a los machos (Domínguez-Roldan et al. 2018), y el dimorfismo de la infección afecta la prolificidad, al reducir a la mitad la camada de hembras infectadas con cisticercos de T. pisiformis respecto a camadas de conejas sanas (Hallal-Calleros et al. 2016); esto se traduce en pérdidas económicas por esta parasitosis en la producción cunícola.

Estudios sobre $T$. pisiformis consideran este parásito como modelo de estudio en otras cestodiasis de importancia en la salud pública y animal, como lo son T. solium L. y T. saginata Goeze (Toral-Bastida et al. 2011), y la similitud antigénica y proteica que comparten T. pisiformis con T. hydatigena Pallas y Echinococcus granulosus (Batsch) lo hacen una especie modelo para el estudio de céstodos notificados como zoonosis (Liu et al. 1992). Para el control del "complejo Taenia-cisticercosis" por T. solium en humanos, uno de los fármacos mayormente empleados es albendazol (ABZ) (Prasad y Singh 2018); sin embargo, ABZ causa efectos secundarios como náuseas y vómito, además de ser hepatotóxico y teratogénico. Asimismo, la permanencia de ABZ en el ambiente es prolongada; afecta al ecosistema al ser tóxico para organismos como la lombriz Eisenia fetida (Savigny) a una concentración de $600 \mathrm{mg} \mathrm{kg}^{-1}$, en un lapso de 7 a 14 días, mientras que $1 \mu \mathrm{M}$ de $\mathrm{ABZ}$ causa malformaciones en embriones de pez cebra, Danio rerio Hamilton, en un lapso de 48 h (Gao et al. 2007; Carlsson et al. 2011).

El empleo de hongos nematófagos ha sido una estrategia de control para la fase de huevo de $T$. saginata y T. taeniaiformis (Batsch) (Araújo et al. 2009;
Braga et al. 2009); por lo tanto, el uso de antagonistas naturales se debe considerar como una alternativa ecológica para el control de Taenia spp.

La proteína Cry5B de Bacillus thuringiensis Berliner ha sido empleada en el control de nemátodos parásitos como Ancylostoma ceylanicum Looss y Ascaris suum Goeze, con elevada actividad nematicida in vitro (Cappello et al. 2006; Urban et al. 2013). La bacteria $B$. thuringiensis produce, además de proteínas Cry, otros factores de virulencia, como metaloproteasas y quitinasas relacionadas con la actividad nematicida (Ruan et al. 2015). Se han referido estudios de patogenicidad de la bacteria en contra de nemátodos de vida libre, como Caenorhabditis elegans (Maupas), y sobre nemátodos fitopatógenos, como Meloidogyne sp. (Zhang et al. 2012; Zhang et al. 2016; Abbasi et al. 2019). Entre los signos de patogenicidad de B. thuringiensis sobre $C$. elegans, la disminución de tamaño es un indicador de toxicidad (Zhang et al. 2016), mientras que los signos de patogenicidad en parásitos tratados con la cepa GP526 de B. thuringiensis en el céstodo Dipylidium caninum L. son la disminución de la motilidad, daño sobre el tegumento y actividad ovicida (Peña et al. 2013); por su parte, en el tremátodo Centrocestus formosanus (Nishigori), GP526 induce la destrucción caudal del parásito (Mendoza-Estrada et al. 2016).

En evaluaciones clínicas con antihelmínticos convencionales para el control de céstodos y nemátodos parásitos de animales, la carga de huevos en excretas (huevos por gramo de heces) es un parámetro ampliamente utilizado para el diagnóstico y seguimiento de helmintiasis. Además, la liberación de la fase infectiva en huevos de Taenia sp. es un factor importante en la propagación de la cisticercosis (Khan et al. 2019). El presente trabajo propone el uso de la cepa GP526 como candidato para controlar parasitosis causadas por céstodos como T. pisiformis

\section{Materiales Y MÉtodos}

\section{Obtención del complejo espora-cristal}

Se utilizó la cepa GP526, perteneciente al laboratorio de Parasitología Vegetal del Centro de Investigaciones Biológicas de la Universidad Autónoma del Estado de 
Morelos. La cepa fue activada en placas de Petri, con medio específico de esporulación (HCT), durante 96 $\mathrm{h}$, hasta su esporulación completa, y el complejo espora-cristal de la biomasa bacteriana fue recuperado con un asa bacteriológica en agua destilada estéril. La cuantificación de la proteína total del complejo espora-cristal se llevó a cabo mediante el método de Bradford (Bradford 1976).

\section{Obtención de los parásitos}

Se obtuvieron 36 proglótidos de T. pisiformis de entre 0.8 y $1.2 \mathrm{~cm}$ de longitud, y la identificación del céstodo adulto se hizo bajo el criterio de morfometría de ganchos descrita por Gubányi (1995). Los proglótidos fueron enjuagados con solución salina a $0.9 \%$, y los proglótidos grávidos fueron cortados del céstodo adulto con bisturí, en zonas entre segmentos sin prolongación uterina. Los proglótidos cortados fueron sellados por presión mecánica con pinzas de disección en los extremos, para evitar la salida de los huevos; cada proglótido presentó un poro genital. Los segmentos se aleatorizaron por grupo y tratamiento.

\section{Efecto de GP526 sobre proglótidos grávidos de $T$. pisiformis}

Para la evaluación del efecto de GP526 sobre la expulsión de huevos por proglótido, se efectuaron ensayos de expulsión mecánica inducida mediante la siguiente metodología: cada proglótido fue colocado individualmente en tubos Eppendorf de $1.5 \mathrm{~mL}$ y divididos en grupos de tres proglótidos por tratamiento. Los tratamientos tuvieron un control negativo con agua destilada estéril, $0.1 \mathrm{mg} / \mathrm{mL}$ de proteína del complejo espora-cristal de GP526 o control positivo con albendazol (ABZ) a $4 \mathrm{mg} / \mathrm{mL}$. Los proglótidos fueron mantenidos en incubación a $37^{\circ} \mathrm{C}$ a 1,100 rpm en Thermomixter 5436b. La lectura de los huevos liberados se llevó a cabo con $10 \mu \mathrm{L}$, y el cálculo de huevos fue realizado para un volumen de $1 \mathrm{~mL}$ por proglótido cada $30 \mathrm{~min}$, durante 2.5 $\mathrm{h}$, tiempo en el que el grupo control mantuvo la liberación de huevos por el poro genital bajo estas condiciones. Los ensayos fueron repetidos en tres ocasiones, por triplicado de cada tratamiento.

\section{Análisis morfotisular de proglótidos pretratados}

Se fotodocumentó el daño causado sobre los proglótidos maduros en tres tratamientos experimentales: 1) control negativo: medio RPMI-1640; 2) GP526: $0.1 \mathrm{mg} /$ $\mathrm{mL}$ del complejo espora-cristal de GP526, y 3) control positivo: $\mathrm{ABZ}$ a $4 \mathrm{mg} / \mathrm{mL}$, a temperatura ambiente $\left(27^{\circ} \mathrm{C}\right)$ en medio RPMI-1640 durante $96 \mathrm{~h}$. El ensayo se efectuó con tres proglótidos por tratamiento, y los proglótidos fueron fijados en paraformaldehido a $4 \%$ durante $24 \mathrm{~h}$ a $4^{\circ} \mathrm{C}$. Posteriormente fueron teñidos con Hematoxilina de Harris. Asimismo, los proglótidos evaluados en el ensayo de la expulsión mecánica de huevos de los grupos control, GP526 y ABZ fueron enjuagados con agua destilada estéril y posteriormente teñidos con Safranina a 5\%.

\section{Análisis estadísticos}

Para el análisis de la liberación de los huevos por tratamiento, así como la comparación de liberación de huevos en el grupo control a diferentes periodos de incubación, se hicieron pruebas de ANOVA y la prueba paramétrica de Tukey-Kramer con un nivel de confianza de $95 \%$ en cada caso.

\section{Resultados y discusión}

Los resultados tisulares indicaron que los poros genitales del control no presentaron alteraciones (Figura 1A), en tanto que los poros genitales de proglótidos maduros experimentaron cambios morfológicos al ser incubados con GP526 a concentración de $0.1 \mathrm{mg} / \mathrm{mL}$, al generar contracción del tegumento después de 96 $\mathrm{h}$ de incubación a $27^{\circ} \mathrm{C}$ (Figura 1B). Un efecto similar se obtuvo en proglótidos tratados con ABZ (Figura 1C). Las observaciones en el tegumento coinciden con lo referido por Peña et al. (2013), quienes reportaron alteración en el tegumento del céstodo $D$. caninum tratado con la cepa GP526.

El tratamiento con proglótidos grávidos a $37^{\circ} \mathrm{C}$ con agitación de 1,100 rpm permitió la liberación de huevos por el poro genital; se observó menor liberación de huevos en proglótidos tratados con ABZ desde las $0.5 \mathrm{~h}$ post incubación (Figura $2 \mathrm{~A}$ y B), mientras que la 

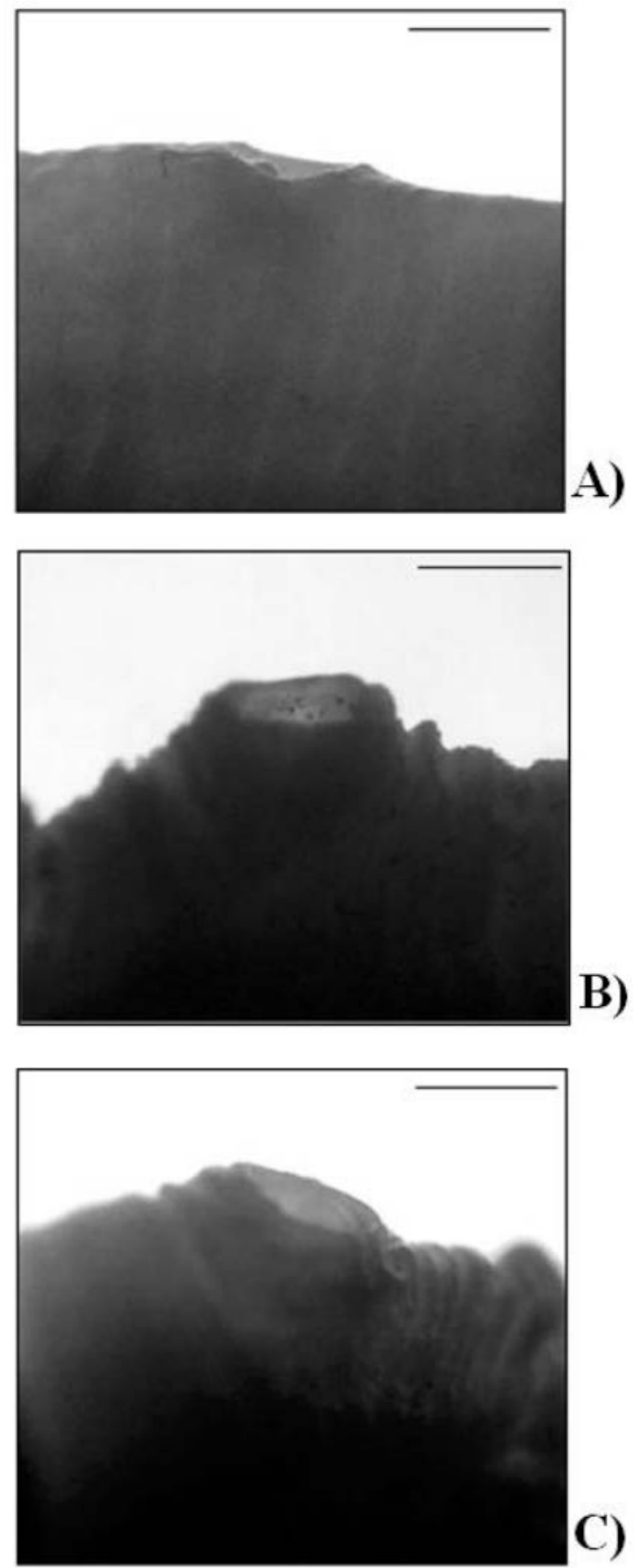

Figura 1. Poros genitales de proglótidos maduros de T. pisiformis. Se ilustra la zona afectada por tratamientos in vitro durante $96 \mathrm{~h}$ a $27^{\circ} \mathrm{C}$ con GP526 o ABZ en comparación con el poro genital del grupo control (A), poro genital post tratamiento con GP526 $0.1 \mathrm{mg} / \mathrm{mL}$ (B) y poro genital post tratamiento con ABZ a $4 \mathrm{mg} / \mathrm{mL}(\mathrm{C})$, Barras $=1 \mathrm{~mm}$. disminución en la liberación de huevos inducida por GP526 pudo observarse a partir de $1.5 \mathrm{~h}$ de incubación (Figura 2C- E). La liberación de huevos en el grupo control se mantuvo continua y en incremento hasta las $2.5 \mathrm{~h}$ de incubación (Figura 2E); este fenómeno posiblemente se deba a que la velocidad de agitación permitió un número limitado de huevos localizados en la cercanía del poro genital.

Hasta el momento, existe poca información del efecto de agentes cestocidas sobre la liberación de huevos de Taenia sp. in vitro, por lo que este trabajo brinda un acercamiento de cómo evaluar este evento biológico. Se sabe que los huevos de Taenia sp. pueden liberarse al ambiente fuera del proglótido o dentro del mismo, y que algunos segmentos de parásitos como T. saginata presentan proglótidos mótiles capaces de migrar (Dorny y Praet 2007). Por tanto, los tratamientos con agentes cestocidas que causan la alteración del tegumento o de la musculatura en el proglótido pueden disminuir su motilidad y la liberación de los huevos. Se debe considerar que la peristalsis intestinal del hospedero puede ayudar mecánicamente a la liberación de los huevos (Maizels y Holland 1998), por lo que en este estudio la incubación de los proglótidos se mantuvo en agitación.

La cepa GP526 indujo una disminución significativa de huevos liberados respecto del grupo control a partir de $1.5 \mathrm{~h}$ post incubación, mientras que ABZ causó una disminución significativa desde las primeras $0.5 \mathrm{~h}$. La disminución de huevos liberados por proglótido puede ser asociada a la disminución de la cantidad de huevos por gramo de heces en humanos teniásicos tratados con ABZ (Khan et al. 2019).

Las evaluaciones durante 0.5 o $1 \mathrm{~h}$ demuestran que GP526 tarda más tiempo en actuar que ABZ y tiene menor efectividad; sin embargo, a $1.5 \mathrm{~h}$ se observa un cambio significativo al disminuir $41.4 \%$ la liberación de huevos en comparación con el control, que liberó en promedio 10,144.4 huevos (Figura 2C); posterior a $2 \mathrm{~h}$ de incubación, causó la disminución de $57.7 \%$ de un promedio de $16,411.1$ huevos obtenidos del control (Figura 2D), y a 2.5 h una disminución de $60.1 \%$ respecto a los $16,711.1$ huevos promedio liberados por el control (Figura 2E). Para ABZ, el rango de efectividad entre 0.5 y $2.5 \mathrm{~h}$ fue de $84.9-92.4 \%$, con un máximo de $97.5 \%$ de disminución a 1.5 h (Figura 
A)

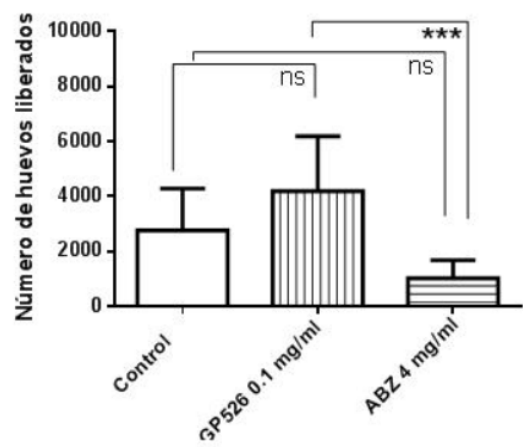

C)

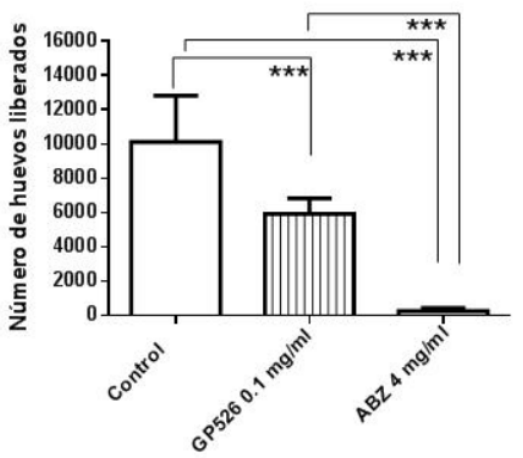

E)

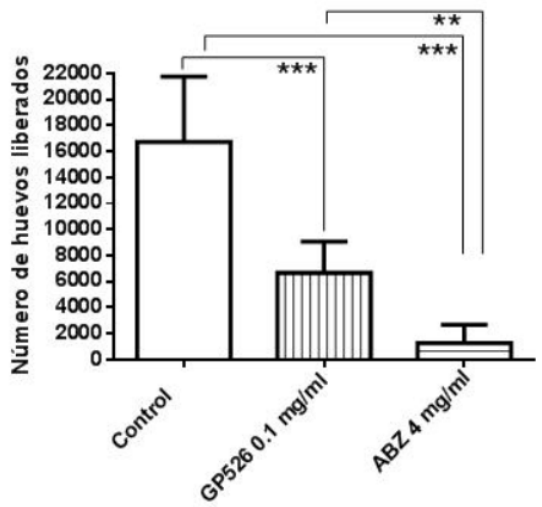

B)

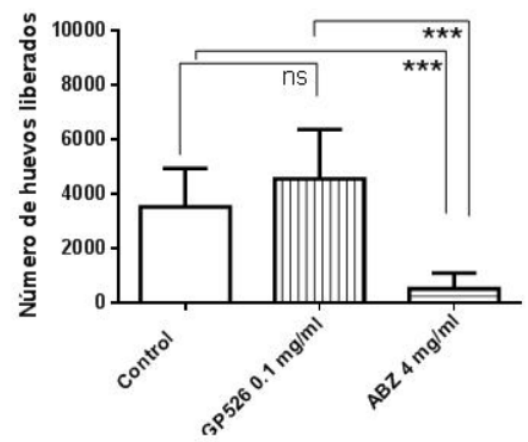

D)

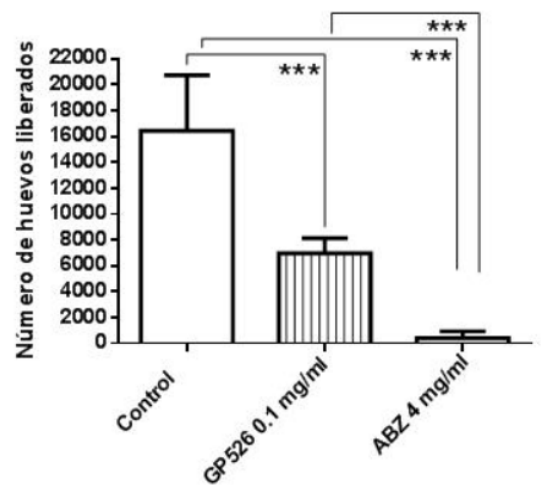

F)

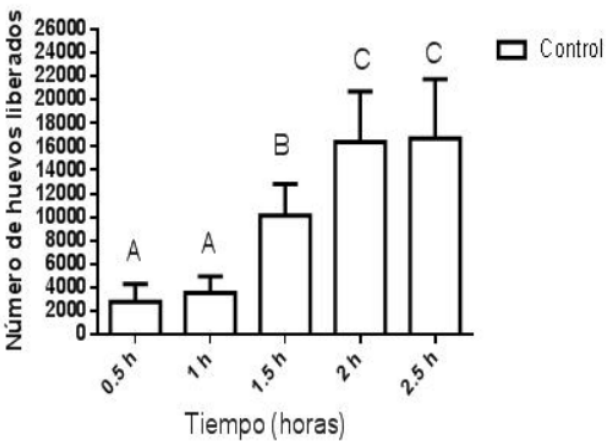

Figura 2. Liberación de huevos de T. pisiformis. Los gráficos muestran promedio \pm desviación estándar de los siguientes tratamientos: GP526 $0.1 \mathrm{mg} / \mathrm{mL}$, ABZ $4 \mathrm{mg} / \mathrm{mL}$ y grupo control, durante $0.5 \mathrm{~h}(\mathrm{~A}), 1 \mathrm{~h}(\mathrm{~B}), 1.5 \mathrm{~h}(\mathrm{C}), 2 \mathrm{~h}(\mathrm{D}), 2.5 \mathrm{~h}(\mathrm{E}), \mathrm{y}$ la liberación de los huevos del grupo control a los diferentes tiempos de incubación (0.5 a $2.5 \mathrm{~h})$ (F). Literales diferentes muestran diferencia estadística mediante Tukey-Kramer $(\alpha=0.05)$; ns $=$ no significativo, ${ }^{* *} \mathrm{P}<0.01,{ }^{* * *} \mathrm{P}<0.001$.

2A-E), mientras que el grupo control se mantuvo estable en evaluaciones a 2 y $2.5 \mathrm{~h}$ (Figura $2 \mathrm{~F}$ ). En el presente estudio fue notable una mayor efectividad de ABZ respecto a GP526 en todas las evaluaciones efectuadas.

La actividad de disminución de los huevos liberados por la acción de GP526 a 1.5 h se considera de acción rápida, al contrastar que la acción de la bacteria $B$. thuringiensis sobre nemátodos supera las $12 \mathrm{~h}$ de interacción (Cappello et al. 2006). El efecto de GP526 en horas se notificó en la actividad ovicida del complejo espora-cristal sobre huevos de $D$. caninum, con un tiempo de $3.5 \mathrm{~h}$ post tratamiento y una efectividad máxima de $82.7 \%$ a concentración de $1 \mathrm{mg} / \mathrm{mL}$ de proteína, así como la disminución de la motilidad del céstodo en $70 \%$ a $2 \mathrm{~h}$ post tratamiento 
(Peña et al. 2013), lo que coincide con el tiempo de acción sobre proglótidos grávidos de T. pisiformis. Sin embargo, para afectar la motilidad del céstodo $D$. caninum fue necesaria una concentración 100 veces mayor a la empleada en este estudio para T. pisiformis.

El conteo de huevos liberados por gramo de heces es un criterio que se ha usado para evaluar la eficacia de ABZ (Khan et al. 2019); los hallazgos sobre la cantidad de huevos liberados por proglótidos tratados con $\mathrm{ABZ}$ sugieren que podría haber una disminución de la cantidad de huevos liberados por daño en los proglótidos sin necesidad de la expulsión de la tenia, y, por consiguiente, encontrar un menor número de huevos en estudios coproparasitológicos, pues en los tratamientos con ABZ para la teniasis en humanos es necesaria la administración de $400 \mathrm{mg}$ del fármaco hasta por 3 días para la expulsión del parásito (Misra et al. 1984; Ash et al. 2017).

La disminución en el número de huevos liberados por proglótidos post tratamiento en menos de tres horas resulta de interés, ya que en los céstodos de Taenia sp., como T. asiatica Eom \& Rim, pueden desarrollar más de 1,000 proglótidos (Verster 1969). Al proponer que GP526 es capaz de disminuir la liberación de huevos por proglótido de T. pisiformis se ayuda a contemplar a la cepa como posible tratamiento profiláctico desde el hospedero definitivo, con la finalidad de disminuir la diseminación de la fase infectante del huevo, y, por consiguiente, disminuir la frecuencia de cisticercos en conejos, es decir, controlar la parasitosis del segundo hospedero (hospedero intermediario), al reducir la liberación de huevos en el primer hospedero (hospedero definitivo). Por tanto, la aplicación de GP526 en campo para el control del céstodo se basaría en la implementación de la proteína de GP526 en bebederos cebo para cánidos silvestres y domésticos positivos a T. pisiformis, en lugares estratégicos como cultivos de alfalfa y producciones cunícolas de traspatio, con el objetivo de anticipar la alteración de los proglótidos en el hospedero antes de su expulsión mediante la defecación. Sin embargo, futuras investigaciones enfocadas en el tratamiento de teniasis son necesarias para evaluar la disminución de huevos en estudios coproparasitoscópicos en perros infectados con T. pisiformis tratados con GP526.

En España, específicamente en la región de Cataluña, se han cuantificado las pérdidas económicas anuales a causa de las canales infectadas por T. saginata, que son de $19,442 €$, y la erogación por concepto de inspección de las canales bovinas de 127,566 € (Laranjo-González et al. 2018), por lo que la cisticercosis causada por $T$. saginata genera importantes pérdidas económicas. El impacto económico de $T$. pisiformis es desconocido; no obstante, otra tenia relacionada con el hospedero definitivo de T. pisiformis es T. ovis (Cobbold). Se ha notificado que dicho parásito afecta la economía de granjas de ovinos, al presentar infección en más de la mitad de las ovejas de una granja ubicada en la provincia de Gansu, al noroeste de China, con una pérdida económica de hasta 16,000 dólares en 2015 (Shi et al. 2016); por tanto, el control de la fase infectante del huevo es un factor importante para disminuir las pérdidas económicas atribuibles al decomiso de canales infectadas.

En los proglótidos control fue posible observar la disminución de huevos en ramas uterinas y su salida a través del poro genital (Figura 3A), mientras que los proglótidos tratados con GP526 presentan el conjunto de huevos en ramas uterinas cercanas a la periferia del proglótido, sin afectar el conducto que comunica la zona uterina con el poro genital (Figura 3B); esto sugiere que GP526 no afecta la salida de los huevos por el conducto, en comparación con proglótidos grávidos tratados con $\mathrm{ABZ}$, en donde el conjunto de huevos es observado dentro de las ramas uterinas del proglótido (Figura 3C), evidencia de que el fármaco afecta también el conducto que comunica las ramas uterinas con el poro genital.

Cada segmento maduro de Taenia sp. cuenta con un poro genital en el borde lateral (Baer 1956); también se ha referido que $T$. saginata puede tener hasta dos poros genitales funcionales (Merdivenci 1964) y la atrofia de esta zona provoca una disminución en la funcionalidad del proglótido que se traduce en un menor número de huevos liberados.

En el presente artículo, se aportan las bases experimentales in vitro para proponer una estrategia de control útil basada en el uso de la cepa GP526, cuyo objetivo es alterar la fisiología de la liberación de huevos y del tegumento del poro genital en proglótidos grávidos de T. pisiformis, para interrumpir la capacidad infectiva del parásito. 

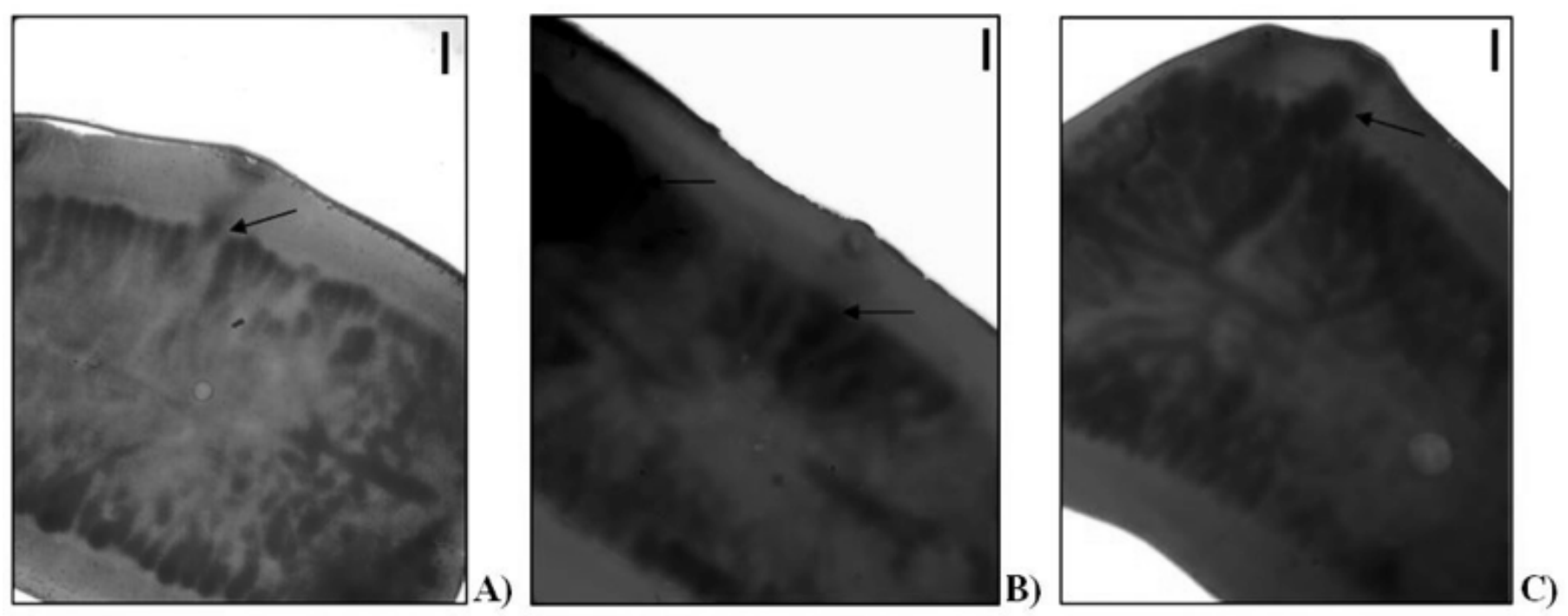

Figura 3. Proglótidos grávidos de T. pisiformis. Los proglótidos fueron tratados con GP526 y ABZ respecto al proglótido control después de 2.5 h de incubación a $37^{\circ}$ C a 1,100 rpm. Proglótido control (A), la flecha ilustra la fluidez de los huevos en el conducto, en el interior del proglótido, y ramificaciones uterinas con baja carga de huevos en proglótido. Proglótido tratado con $0.1 \mathrm{mg} / \mathrm{mL}$ de GP526 (B), las flechas muestran los sitios donde los huevos presentaron acumulación. Se observa una carga considerable de huevos dentro del proglótido tratado con $4 \mathrm{mg} / \mathrm{mL}$ de $\mathrm{ABZ}$ (C), la flecha muestra la acumulación de los huevos en la zona del conducto cercano al poro genital, con ramas uterinas repletas de huevos. Barras $=1 \mathrm{~mm}$.

\section{Conclusiones}

El complejo espora-cristal de GP526 afecta el tegumento del poro genital de T. pisiformis in vitro, además de causar la disminución de huevos liberados por el proglótido en comparación con los huevos liberados por proglótidos $\sin$ B. thuringiensis, así como la retención de huevos en los proglótidos al ser tratados con GP526 o ABZ. Por tanto, ambos tratamientos pueden considerarse efectivos para disminuir la propagación de los huevos de T. pisiformis.

\section{Agradecimientos}

EDG agradece al Consejo Nacional de Ciencia y Tecnología (CONACyT) por el apoyo otorgado (305290). 


\section{LITERATURA CITADA}

Abbasi MW, Khan MQ, Zaki MJ, Shaukat SS, Rauf A, Ahmed N, Tariq M. 2019. Soil application of Bacillus thuringiensis Berliner isolates against root-knot nematode (Meloidogyne javanica (Treub) Chitwood) in okra (Abelmoschus esculentus (L.) Moench). Acta Phytopathologica et Entomologica Hungarica 54: 173186. http://doi.org/10.1556/038.54.2019.019

Araújo JM, Araújo JV, Braga FR, Carvalho RO, Silva AR, Campos AK. 2009. Interaction and ovicidal activity of nematophagous fungus Pochonia chlamydosporia on Taenia saginata eggs. Experimental Parasitology 121: 338-341. https://doi.org/10.1016/j.exppara.2008.12.011

Ash A, Okello A, Khamlome B, Inthavong P, Allen J, Thompson RCA. 2017. Controlling Taenia solium and soil transmitted helminths in a northern Lao PDR village: Impact of a triple dose albendazole regime. Acta Tropica 174: 171-178. https://doi.org/10.1016/j. actatropica.2015.05.018

Baer JG. 1956. The taxonomic position of Taenia madagascariensis Davaine, 1870, a tapeworm parasite of man and rodents. Annals of Tropical Medicine \& Parasitology 50: 152-156. https://doi.org/10.1080/00034983.1956.11685754

Bradford MM. 1976. A rapid and sensitive method for the quantitation of microgram quantities of protein utilizing the principle of protein-dye binding. Analytical Biochemistry 72: 248-254. https://doi. org/10.1016/0003-2697(76)90527-3

Braga FR, Araújo JV, Carvalho RO, Silva AR, Araujo JM, Tavela AO, Campos PRS, Campos AK. 2009. Ovicidal effect of nematophagous fungi on Taenia taeniaeformis eggs. World Journal of Microbiology and Biotechnology 25: 533-535. https://doi.org/10.1007/s11274-008-9920-2

Cappello M, Bungiro RD, Harrison LM, Bischof LJ, Griffitts JS, Barrows BD, Aroian RV. 2006. A purified Bacillus thuringiensis crystal protein with therapeutic activity against the hookworm parasite Ancylostoma ceylanicum. Proceedings of the National Academy of Sciences 103: 15154-15159. https://doi.org/10.1073/pnas.0607002103

Carlsson G, Patring J, Ullerås E, Oskarsson A. 2011. Developmental toxicity of albendazole and its three main metabolites in zebrafish embryos. Reproductive Toxicology 32: 129-137. https://doi.org/10.1016/j. reprotox.2011.05.015

Coman BJ, Rickard MD. 1975. The location of Taenia pisiformis, Taenia ovis and Taenia hydatigena in the gut of the dog and its effect on net environmental contamination with ova. Zeitschrift für Parasitenkunde 47: 237248. https://doi.org/10.1007/BF00389883

DeWolf BD, Peregrine AS, Jones-Bitton A, Jansen JT, Menzies PI. 2014. Taenia ovis infection and its control: A Canadian perspective. New Zealand Veterinary Journal 62: 1-7. https://doi.org/10.1080/00480169.2013 .832109

Domínguez-Roldan R, Pérez-Martínez M, Rosetti MF, Arias-Hernández D, Bernal-Fernández G, FloresPérez FI, Hallal-Calleros C. 2018. High frequency of Taenia pisiformis metacestodes and high sex-associated susceptibility to cysticercosis in naturally infected wild rabbits. Parasitology Research 117: 2201-2206. https://doi.org/10.1007/s00436-018-5907-6

Dorny P, Praet N. 2007. Taenia saginata in Europe. Veterinary Parasitology 149: 22-24. https://doi. org/10.1016/j.vetpar.2007.07.004

Gao Y, Sun Z, Sun X, Sun Y, Shi W. 2007. Toxic effects of albendazole on adenosine triphosphatase activity and ultrastructure in Eisenia fetida. Ecotoxicology and Environmental Safety 67: 378-384. https://doi. org/10.1016/j.ecoenv.2006.10.008

Gubányi A. 1995. Morphometrics of taeniid tapeworms I. Multivariate analysis of distance measurements of the rostellar hooks. Parasitologia Hungarica 28: 21-41.

Hallal-Calleros C, Morales-Montor J, Orihuela-Trujillo A, Togno-Peirce C, Murcia-Mejía C, Bielli A, Hoffman KL, Flores-Pérez FI. 2016. Taenia pisiformis cysticercosis induces decreased prolificacy and increased progesterone levels in rabbits. Veterinary Parasitology 229: 50-53. https://doi.org/10.1016/j.vetpar.2016.09.015

Khan W, Iqbal M, Khan I. 2019. Albendazole in the treatment of ancylostomiasis, ascariasis, taeniasis and amoebiasis in school children. Pakistan Journal of Zoology 51: 1587-1587. https://doi.org 10.17582/journal.pjz/2019.51.4.sc4

Laranjo-González M, Devleesschauwer B, Jansen F, Dorny P, Dupuy C, Requena-Méndez A, Allepuz A. 2018. Epidemiology and economic impact of bovine cysticercosis and taeniosis caused by Taenia saginata in northeastern Spain (Catalonia). Parasites \& Vectors 11: 376. https://doi.org/10.1186/s13071-018-2931-4

Liu D, Rickard MD, Lightowlers MW. 1992. Comparative immunoelectrophoretic analysis of Echinococcus granulosus, Taenia hydatigena and Taenia pisiformis cyst fluid antigens by hyperimmune rabbit sera. 
Research in Veterinary Science 53: 133-135. https:// doi.org/10.1016/0034-5288(92)90099-N

Loos-Frank B. 2000. An up-date of Verster's (1969) 'Taxonomic revision of the genus Taenia Linnaeus' (Cestoda) in table format. Systematic Parasitology 45: 155-184. https://doi.org/10.1023/A:1006219625792

MaizelsRM,Holland MJ.1998.Parasiteimmunity:Pathways for expelling intestinal helminths. Current Biology 8: 711-714. https://doi.org/10.1016/S0960-9822(98)70455-5

Mendoza-Estrada LJ, Hernández-Velázquez VM, ArenasSosa I, Flores-Pérez FI, Morales-Montor J, Peña-Chora G. 2016. Anthelmintic effect of Bacillus thuringiensis strains against the gill fish trematode Centrocestus formosanus. BioMed Research International 2016: 8272407. https://doi.org/10.1155/2016/8272407

Merdivenci A. 1964. An abnormal Taenia saginata with double genital pores. Journal of Parasitology 50: 476-477.

Misra RC, Dewan R, Jagota SC. 1984. Treatment of human taeniasis with albendazole. Current Therapeutic Research 36: 1195-1197.

Peña G, Aguilar F, Hallal-Calleros C, Morales-Montor J, Hernández-Velázquez VM, Flores-Pérez FI. 2013. In vitro ovicidal and cestocidal effects of toxins from Bacillus thuringiensis on the canine and human parasite Dipylidium caninum. BioMed Research International 2013: 174619. https://doi.org/10.1155/2013/174619

Prasad KN, Singh SK. 2018. Taeniasis and neurocysticercosis: Emerging public health problems. En: Singh P, editor. Infectious Diseases and Your Health. Singapur, Springer. P. 113-134.

Ruan L, Crickmore N, Peng D, Sun M. 2015. Are nematodes a missing link in the confounded ecology of the entomopathogen Bacillus thuringiensis? Trends in Microbiology 23: 341-346. https://doi.org/10.1016/j.tim.2015.02.011

Shi W, He W, Guo X, Liu Q, Gao S, Zhan F, Liu X, Pan Y, Luo X, Zheng Y. 2016. The first outbreak of Taenia ovis infection in China. Parasitology International 65: 422423. https://doi.org/10.1016/j.parint.2016.06.005

Toral-Bastida E, Garza-Rodriguez A, Jimenez-Gonzalez DE, Garcia-Cortes R, Avila-Ramirez G, Maravilla P, Flisser A. 2011. Development of Taenia pisiformis in golden hamster (Mesocricetus auratus). Parasites \& Vectors 4: 147. https://doi.org/10.1186/1756-3305-4-147

Urban JFJr, Hu Y, Miller MM, Scheib U, Yiu YY, Aroian RV. 2013. Bacillus thuringiensis-derived Cry5B has potent anthelmintic activity against Ascaris suum. PLoS Neglected Tropical Diseases 7: e2263. https://doi.
org/10.1371\%2Fjournal.pntd.0002263

Verster A. 1969. A taxonomic revision of the genus Taenia Linnaeus, 1758 S. str. Onderstepoort Journal of Veterinary Research 36: 3-58.

Zhang F, Peng D, Ye X, Yu Z, Hu Z, Ruan L, Sun M. 2012. In vitro uptake of $140 \mathrm{kDa}$ Bacillus thuringiensis nematicidal crystal proteins by the second stage juvenile of Meloidogyne hapla. PLoS ONE 7: e38534. https://doi. org/10.1371/journal.pone.0038534

Zhang F, Peng D, Cheng C, Zhou W, Ju S, Wan D, Yu Z, Shi J, Deng Y, Wang F, Ye X, Hu Z, Lin J, Ruan L, Sun M. 2016. Bacillus thuringiensis crystal protein Cry6Aa triggers Caenorhabditis elegans necrosis pathway mediated by aspartic protease (ASP-1). PLoS Pathogens 12: e1005389. https://doi.org/10.1371/journal.ppat.1005389 